\title{
Fatores associados à sífilis congênita em um hospital público no estado do Rio de Janeiro, Brasil
}

\section{Factors associated with congenital syphilis in a public hospital on the state of Rio de Janeiro, Brazil}

\author{
Christiane Fernandes Ribeiro, ${ }^{1}$ Matheus Nascimento da Silva, ${ }^{1}$ Layla Couto Araujo, ${ }^{1}$ Thais Perroni El Saman ${ }^{1}$ e \\ Maria Isabel do Nascimento ${ }^{1}$
}

'Universidade Federal Fluminense

\section{Palavras-chave:}

Sífilis congênita; Cuidado pré-natal; Assistência perinatal; Modelos teóricos; Análise de regressão.

\section{Resumo}

Introdução: a sífilis é uma doença infecciosa, de transmissão sexual, transplacentária ou transfusional, que pode progredir para aborto, morte fetal, prematuridade, baixo peso ao nascer e morte neonatal. Objetivo: avaliar as características de mães de bebês nascidos vivos com sífilis congênita. Métodos: todas as mães de bebês $(n=$ 46) nascidos a termo que receberam tratamento de sífilis congênita, no período neonatal, de $1{ }^{\circ}$ de outubro de 2014 a 30 de setembro de 2015, foram incluídas no estudo e comparadas com mães de bebês (n = 157) sem sífilis congênita. Resultados: a regressão de Poisson com ajuste robusto de variância verificou as associações com o desfecho sífilis congênita, bem como as razões de prevalência e intervalos de confiança de $95 \%$. O principal fator de risco de sífilis congênita foi a baixa escolaridade materna (RP ajustada: 1,93; IC 95\%: 1,056-3,526). Os fatores protetores foram o pré-natal na cidade de Niterói (RP ajustada: 0,48; IC 95\%: 0,253-0,911) e ter tido pelo menos três testes laboratoriais para sífilis (RP ajustada: 0,35; IC 95\%: 0,183-0,686). Conclusões: a baixa escolaridade foi um fator de risco importante para a sífilis congênita e o efeito protetor, o número de testes de sífilis realizados.

\section{Keywords:}

Syphilis, congenital; Prenatal care; Perinatal care; Models, theoretical; Regression analysis.

\footnotetext{
Abstract

Introduction: syphilis is an infectious disease, of sexual, transplacental and transfusional transmission that can progress to miscarriage, fetal death, prematurity, low birth weight, and neonatal death. Objective: to analyze the characteristics of mothers of newborns with congenital syphilis. Methods: all mothers of term newborns diagnosed with congenital syphilis $(n=46)$ and treated during the neonatal period from October 1, 2014 through September 30, 2015 were included and compared with mothers of babies without congenital syphilis ( $n=157)$. The Poisson regression with robust adjustment of the variance verified the associations and estimated prevalence ratios and 95\% confidence intervals. Results: the main risk factor was low education level (adjusted PR: 1.93; 95\% Cl: 1.056-3.526). The protection factors were prenatal care in the city of Niterói, RJ, Brazil (adjusted PR: 0.48; 95\% Cl: 0.253-0.911) and having at least three syphilis laboratory tests (adjusted PR: 0.35; 95\% Cl: 0.183-0.686). Conclusions: the low education level remains a main risk factor for congenital syphilis. However, a major protective effect was determined by the number of syphilis laboratory tests.
} 


\section{INTRODUÇÃO}

A sífilis é uma doença infecciosa polimorfa de transmissão eminentemente sexual, podendo de ser adquirida também por passagem transplacentária e por via transfusional. ${ }^{1}$ A transmissão de mãe para feto determina resultados adversos com graus diferenciados, mesmo em gestantes que têm acesso ao pré-natal. ${ }^{2,3}$ Os desfechos de sífilis congênita incluem abortamento, óbito fetal, prematuridade, baixo peso ao nascer e óbito neonatal em proporções diferenciadas tanto na presença quanto na ausência de tratamento. ${ }^{4}$

Embora o diagnóstico e o tratamento da sífilis materna e adquirida transcorram com relativa facilidade, o da sífilis congênita no período neonatal exige criteriosa avaliação epidemiológica da situação materna, bem como interpretação clinicolaboratorial cuidadosa do estado da criança, estudo de imagem e procedimentos invasivos de punção de liquor. ${ }^{5}$

Apesar dos recursos disponíveis para controlar a sífilis, a doença permanece entre os principais problemas de saúde pública no mundo. A Organização Mundial da Saúde (OMS) estimou que em 2013 havia 1,9 milhões de gestantes infectadas no mundo, cobertura menor que $10 \%$ de diagnóstico e tratamento durante a gestação e frequência de resultados adversos variando de $53,4 \%$ a $88,1 \% .{ }^{6}$

O Brasil está relacionado entre os 15 países considerados prioritários para o controle da sífilis congênita, em função da prevalência da doença na gestação e do tamanho da população brasileira. Os indicadores produzidos a partir de dados fornecidos pelo país e publicados pela OMS mostraram a prevalência de $1,6 \%$ e de $1,1 \%$ de positividade para sífilis entre gestantes que frequentaram o pré-natal em 2008 e em 2011, respectivamente. O documento também mostrou uma variação de taxas de sífilis congênita de 165,7 para 220,9 por 100 mil nascidos vivos de 2007 para 2010 . $^{7}$

Este panorama indica que as estimativas produzidas a partir de notificações feitas pelo município de Niterói, no Rio de Janeiro, tendem ao distanciamento dos parâmetros $(<0,5 / 1.000$ nascidos vivos) indicativos de controle de sífilis congênita propostos pela OMS. ${ }^{7}$

Considerando a morbimortalidade decorrente da sífilis congênita e a necessidade de contribuições em direção ao seu controle, o objetivo do presente estudo foi analisar as características de mães de bebês com sífilis congênita e possíveis fatores de risco.

\section{MÉTODO}

A seguir, explicitaremos brevemente o método empregado no desenvolvimento da presente pesquisa.

\section{CENÁRIO DO ESTUDO}

Este estudo foi desenvolvido na maternidade do Hospital Estadual Azevedo Lima (HEAL), uma unidade hospitalar de média e alta complexidade, para atendimento de urgência e emergência por demanda espontânea e referenciada e atendimento de gestação de alto risco, da região Metropolitana II do estado do Rio de Janeiro.

\section{PARTICIPANTES}

Foram selecionadas mães e recém-nascidos a termo, no período de outubro de 2014 a setembro de 2015 formando dois grupos: grupo com sífilis congênita (grupo SC) e grupo sem sífilis congênita (grupo SSC).

Critério de inclusão: foram consideradas adequadas para o grupo SC as mães cujos recém-nascidos estavam em tratamento parenteral com penicilina G cristalina. O grupo SSC foi constituído por mães cujos recém-natos não tiveram suspeita clínica ou laboratorial e não receberam qualquer tratamento para sífilis congênita, que se encontravam em alojamento conjunto, no mesmo dia de identificação dos casos de sífilis congênita.

\section{COMPOSIÇÃO DA POPULAÇÃO DE ESTUDO}

Todas as mães que no período da coleta de dados preencheram os critérios para sífilis congênita (grupo SC) foram convidadas para o estudo. Para a composição do grupo de comparação (grupo SSC) foi estabelecida a relação 1:3 e as mães foram selecionadas nos leitos de alojamento conjunto, de modo aleatório, no mesmo dia da inclusão dos casos de sífilis congênita.

\section{COLETA DE DADOS}

A coleta de dados foi feita por meio de entrevista presencial, conduzida por estudantes de medicina e supervisionada pela pesquisadora responsável pelo estudo. Além da entrevista, o cartão da gestante, o prontuário materno e do recém-nato foram consultados para complementar os dados de interesse da pesquisa.

Todas as mães de recém-natos que reuniram as definições de sífilis congênita $(n=56)$ foram convidadas a participar do estudo. Contudo, algumas que se mostraram apreensivas - alegaram preocupação com as condições de saúde do recém-nascido e não aceitaram participar - foram excluídas $(n=10)$. Permaneceram para a análise um total de 203 mães, sendo 46 no grupo SC e 157 no grupo SSC.

\section{VARIÁVEIS DO ESTUDO}

Para a descrição dos casos de sífilis congênita, foram obtidas as seguintes informações: completude do 
tratamento da sífilis materna no pré-natal; tipo de antibiótico usado (penicilina benzatina); momento da gestação que o tratamento foi finalizado; se o parceiro/companheiro foi tratado; e se o tratamento foi acompanhado de práticas sexuais seguras.

Pressupondo que existe uma relação de influência entre os fatores de interesse e o desfecho sífilis congênita, um modelo teórico conceitual ${ }^{8}$ foi concebido considerando três níveis hierárquicos. No nível distal ou sociodemográfico, foram inseridas a idade ( $\leq 19$ anos; 20 a 24 anos; $\geq$ 25 anos) e a escolaridade (fundamental incompleto versus fundamental completo ou mais). No nível intermediário, ligado às características do serviço de saúde, foram analisados o pré-natal em unidades do Programa Médico de Família (PMF) - sim/não; o pré-natal no município de Niterói - sim/não; o pré-natal na rede pública - público exclusivo versus quaisquer outras situações. No nível proximal ou de adequação do pré-natal foram considerados o número de consultas de pré-natal $(<6$ versus $\geq 6$ consultas); e o número de testes de sífilis realizados na gestante/puérpera ( $<3$ testes versus $\geq 3$ testes).

\section{ANÁLISE ESTATÍSTICA}

Empregou-se a regressão de Poisson com estimativa robusta de variância para obter razão de prevalência (RP) bruta e ajustada e respectivos intervalos de confiança (IC) de 95\%. A significância estatística no nível de $5 \%$ foi verificada com o uso do teste de Wald para heterogeneidade.

Primeiramente, foram obtidas estimativas brutas (RP bruta). A seguir, modelos intrablocos foram testados para verificar a influência de cada nível hierárquico, individualmente, no desfecho sífilis congênita. Por fim, variáveis com valor de $\mathrm{p}<0,20$ foram ajustadas, obedecendo a hierarquia do modelo teórico, permanecendo no modelo final os componentes com valor de $\mathrm{p}<0,05$.

A qualidade do ajuste dos modelos (razão de verossimilhança e deviance) e potenciais interações multiplicativas entre os componentes do modelo teórico e o desfecho foram analisadas com o uso do teste qui-quadrado. Todas as análises foram conduzidas com o uso da plataforma R.

\section{ASPECTOS ÉTICOS}

O projeto foi aprovado pelo Comitê de Ética em Pesquisa do Hospital Universitário Antônio Pedro, número CAAE 25342314.1.0000.5243, parecer 790.137, datado de 12 de setembro de 2014. As participantes, devidamente esclarecidas, foram convidadas e entrevistadas somente após a compreensão e assinatura do Termo de Consentimento Livre e Esclarecido.

\section{RESULTADOS}

A média de idade das mães foi de 24,5 anos (desvio-padrão de 6,7 anos). O grupo de mães de bebês com tratamento de sífilis congênita, grupo SC $(22,7 \%)$, foi comparado ao grupo de mães de bebês sem sífilis congênita - grupo SSC (77,3\%). O tratamento completo da sífilis materna no pré-natal foi feito em 41,9\% (18/43) das gestantes, com uso de penicilina benzatina em $27,9 \%$ (12/43), finalizando pelo menos 30 dias antes do parto em $34,9 \%$ (15/43), sendo estendido ao parceiro em $58,1 \%(25 / 43)$ e acompanhado de práticas sexuais seguras durante o tratamento em 45,2\% (19/42).

A comparação da média de idade do grupo SC $(23,1$ anos) e do grupo SSC (24,8 anos) não mostrou diferenças estatisticamente significativas entre as mães. A comparação de frequências dos componentes de cada bloco hierárquico e sífilis congênita apontou para potenciais efeitos de variáveis socioeconômicas do bloco distal (escolaridade), de variáveis relativas aos serviços de saúde do bloco intermediário (pré-natal no PMF e pré-natal em Niterói) e de variáveis de qualidade do pré-natal do bloco proximal (número de testes realizados) como possíveis explicativos do perfil de mães de bebês com sífilis congênita. A distribuição das características analisadas segundo os grupos está apresentada na tabela 1.

A verificação do efeito dos componentes na análise bivariada (RP bruta) mostrou que alguns fatores candidatos (valor de $\mathrm{p}<0,20$ ) à análise multivariada perderam significância estatística na etapa de ajustamento intrabloco. No bloco sociodemográfico, a idade até 19 anos sendo a categoria de referência 25 anos e mais - teve RP bruta de 1,75 (IC 95\%: 0,881-3,467; valor de p: 0,110 ) e RP ajustada por escolaridade de 1,61 (IC 95\%: 0,7903,288; valor de p: 0,189). No bloco sobre características dos serviços de saúde, o pré-natal em unidade do PMF comparado com pré-natal em outro tipo de unidade de saúde teve RP bruta de 0,57 (IC 95\%: 0,309-1,054; valor de p: 0,073) e RP ajustada por pré-natal no município de Niterói de 0,67 (IC 95\%: 0,331-1,342; valor de p: 0,256) (Tabela 2).

Após o ajustamento do modelo final, permaneceram como componentes explicativos de sífilis congênita a baixa escolaridade materna (RP: 1,93; IC 95\%: 1,0563,526), o pré-natal na cidade de Niterói (RP: 0,48; IC 95\%: 0,253-0,911), ter tido pelo menos três testes laboratoriais para sífilis (RP: 0,35; IC 95\%: 0,183-0,686) e ter tido menos de seis consultas de pré-natal (RP: 1,87; IC 95\%: 0,827-4,240). Apesar do componente número de consultas de pré-natal não preencher os critérios estatísticos para a inclusão na análise multivariada (valor de 
Tabela 1 - Distribuição dos componentes dos blocos sociodemográfico (distal), característico dos serviços de saúde (intermediário) e de adequação do pré-natal (proximal) segundo grupos com sífilis e sem sífilis

\begin{tabular}{|c|c|c|c|c|}
\hline Bloco/componente & $\begin{array}{l}\text { Total } \\
\text { n (\%) }\end{array}$ & $\begin{array}{l}\text { Grupo } \\
\text { com sífilis } \\
\text { n (\%) }\end{array}$ & $\begin{array}{l}\text { Grupo } \\
\text { sem sífilis } \\
\text { n(\%) }\end{array}$ & valor de $p$ \\
\hline \multicolumn{5}{|l|}{ Bloco distal } \\
\hline$\underline{\text { Faixa etária }}$ & & & & 0,160 \\
\hline 25 anos ou mais & $83(41,1)$ & $15(32,6)$ & $68(43,6)$ & \\
\hline De 20 a 24 anos & $62(30,7)$ & $13(28,3)$ & $49(31,4)$ & \\
\hline Menos de 20 anos & $57(28,2)$ & $18(39,1)$ & $39(25,0)$ & \\
\hline Escolaridade & & & & 0,013 \\
\hline Fundamental completo & $93(46,0)$ & $28(62,2)$ & $65(41,4)$ & \\
\hline Fundamental incompleto & $109(54,0)$ & $17(37,8)$ & $92(58,6)$ & \\
\hline \multicolumn{5}{|l|}{ Bloco intermediário } \\
\hline Pré-natal no PMF & & & & 0,041 \\
\hline Sim & $125(64,8)$ & $21(51,2)$ & $104(68,4)$ & \\
\hline Não & $68(35,2)$ & $20(48,8)$ & $48(31,8)$ & \\
\hline Pré-natal em Niterói & & & & 0,010 \\
\hline Niterói não & $107(55,4)$ & $15(37,5)$ & $92(60,1)$ & \\
\hline Niterói sim & $86(44,6)$ & $25(62,5)$ & $61(39,9)$ & \\
\hline Pré-natal na rede pública & & & & 0,693 \\
\hline Outras situações & $40(19,7)$ & $10(21,7)$ & $30(19,1)$ & \\
\hline Rede pública exclusiva & $163(80,3)$ & $36(78,3)$ & $127(80,9)$ & \\
\hline \multicolumn{5}{|l|}{ Bloco proximal } \\
\hline$\underline{\text { Número de consultas pré-natal }}$ & & & & 0,218 \\
\hline Menos de 6 & $44(21,7)$ & $13(28,3)$ & $31(19,8)$ & \\
\hline Pelo menos 6 & $159(78,3)$ & $33(71,7)$ & $126(80,2)$ & \\
\hline$\underline{\text { Número de testes de síflis }}$ & & & & 0,001 \\
\hline Menos de 3 & $129(63,9)$ & $19(42,2)$ & $110(70,1)$ & \\
\hline Pelo menos 3 & $73(36,1)$ & $26(57,8)$ & $47(29,9)$ & \\
\hline
\end{tabular}

p: 0,281), optou-se por mantê-lo no modelo final como variável de confusão (Tabela 3 ).

A verificação de potencial interação e da qualidade do ajuste dos modelos estimados mostraram p-valores estatisticamente não significativos, indicando que os modelos foram ajustados adequadamente.

\section{DISCUSSÃO}

Este estudo buscou contribuir com a compreensão do problema da sífilis congênita, uma condição considerada evitável, que ainda persiste entre os principais desafios da área de saúde na região de Niterói e adjacências. As concepções teóricas que nortearam o seu desenvolvimento permitiram caracterizar o perfil de mães de bebês que receberam tratamento de sífilis congênita em uma unidade pública de saúde da Região Metropolitana II, do estado do Rio de Janeiro. A baixa escolaridade aumentou o risco de ter o bebê em tratamento para sífilis congênita. Adicionalmente, ter tido pelo menos três testes para sífilis no período gestacional e o pré-natal em 


\begin{tabular}{|c|c|c|}
\hline Blocos e componentes & $\begin{array}{l}\text { Análise bivariada } \\
\text { RP bruta } \\
\text { (IC 95\%) }\end{array}$ & $\begin{array}{l}\text { Análise ajustada intrabloco } \\
\text { RP ajustada } \\
\text { (IC 95\%) }\end{array}$ \\
\hline \multicolumn{3}{|l|}{ Bloco distal } \\
\hline \multicolumn{3}{|l|}{ Idade } \\
\hline 25 anos ou mais & Ref. & Ref. \\
\hline De 20 a 24 anos & $\begin{array}{l}1,16 \\
(0,552-2,438)\end{array}$ & $\begin{array}{l}1,16 \\
(0,548-2,490)\end{array}$ \\
\hline Menos de 20 anos & $\begin{array}{l}1,75 \\
(0,880-3,467)\end{array}$ & $\begin{array}{l}1,61 \\
(0,790-3,288)\end{array}$ \\
\hline \multicolumn{3}{|l|}{ Escolaridade } \\
\hline Fundamental completo & Ref. & Ref. \\
\hline Fundamental incompleto & $\begin{array}{l}1,93 \\
(1,056-3,526)\end{array}$ & $\begin{array}{l}1,75 \\
(0,949-3,254)\end{array}$ \\
\hline \multicolumn{3}{|l|}{ Bloco intermediário } \\
\hline \multicolumn{3}{|l|}{ Pré-natal no PMF } \\
\hline Não & Ref. & Ref. \\
\hline Sim & $\begin{array}{l}0,57 \\
(0,309-1,053)\end{array}$ & $\begin{array}{l}0,67 \\
(0,331-1,342)\end{array}$ \\
\hline \multicolumn{3}{|l|}{ Pré-natal em Niterói } \\
\hline Niterói não & Ref. & Ref. \\
\hline Niterói sim & $\begin{array}{l}0,48 \\
(0,254-0,914)\end{array}$ & $\begin{array}{l}0,51 \\
(0,244-1,067)\end{array}$ \\
\hline \multicolumn{3}{|l|}{ Pré-natal na rede pública } \\
\hline Sim & Ref. & $x x x$ \\
\hline Não & $\begin{array}{l}1,13 \\
(0,561-2,280)\end{array}$ & $x x x$ \\
\hline \multicolumn{3}{|l|}{ Bloco proximal } \\
\hline \multicolumn{3}{|l|}{ Número de testes de sífilis } \\
\hline Menos de 3 & Ref. & Ref. \\
\hline Pelo menos 3 & $\begin{array}{l}0,41 \\
(0,228-0,747)\end{array}$ & $\begin{array}{l}0,34 \\
(0,180-0,644)\end{array}$ \\
\hline \multicolumn{3}{|c|}{ Número de consultas de pré-natal } \\
\hline Menos de 6 & Ref. & Ref. \\
\hline Pelo menos 6 & $\begin{array}{l}1,42 \\
(0,749-2,704)\end{array}$ & $\begin{array}{l}2,00 \\
(0,986-4,078)\end{array}$ \\
\hline
\end{tabular}

RP - razão de prevalência. IC - intervalo de confiança. Ref. - referência (valor = 1). PMF - Programa Médico de Família.

unidades de saúde de Niterói foram fatores protetores contra a infecção sifilítica.

Com respeito aos aspectos clínicos, a proporção de tratamento completo $(41,9 \%)(18 / 43)$ foi similar à encontrada $(41,8 \%)$ no Distrito Federal no período de 2009 e $2010,{ }^{9}$ mas o tratamento foi encerrado antes dos 30 dias do parto em proporção menor - 34,9\% (15/43) versus
$49,2 \%$. O tratamento do parceiro se consolida como um grande problema que precisa ser devidamente enfrentado, pois a proporção de parceiros não tratados $(58,1 \%-25 / 43)$, na atual casuística, permanece inexplicavelmente alta. Este fato foi previamente constatado por Domingues e Leal nos anos de 2002 (84,6\%), 2003 $(98,0 \%)$ e $2004(100,0 \%){ }^{10}$ 
Tabela 3 - Modelo final de fatores associados à sífilis congênita: análise ajustada segundo componentes do modelo teórico conceitual

\begin{tabular}{|c|c|c|c|}
\hline Blocos e componentes & $\begin{array}{l}\text { Modelo } 1 \\
\text { RP aj. } \\
\text { (IC 95\%) }\end{array}$ & $\begin{array}{l}\text { Modelo } 2 \\
\text { RP aj. } \\
\text { (IC 95\%) }\end{array}$ & $\begin{array}{l}\text { Modelo } 3 \\
\text { Final RP aj. } \\
\text { (IC 95\%) }\end{array}$ \\
\hline \multicolumn{4}{|l|}{ Bloco distal } \\
\hline \multicolumn{4}{|l|}{ Escolaridade } \\
\hline Fundamental completo & Ref. & Ref. & Ref. \\
\hline Fundamental incompleto & $\begin{array}{l}1,93 \\
(1,056-3,526)\end{array}$ & $\begin{array}{l}1,93 \\
(1,056-3,526)\end{array}$ & $\begin{array}{l}1,93 \\
(1,056-3,526)\end{array}$ \\
\hline \multicolumn{4}{|l|}{ Bloco intermediário } \\
\hline \multicolumn{4}{|l|}{ Pré-natal em Niterói } \\
\hline Niterói não & $x x x$ & Ref. & Ref. \\
\hline Niterói sim & $x x x$ & $\begin{array}{l}0,48 \\
(0,253-0,911)\end{array}$ & $\begin{array}{l}0,48 \\
(0,253-0,911)\end{array}$ \\
\hline \multicolumn{4}{|l|}{ Bloco proximal } \\
\hline \multicolumn{4}{|l|}{ Número de testes de sífilis } \\
\hline Menos de 3 & $x x x$ & $x x x$ & Ref. \\
\hline Pelo menos 3 & $x x x$ & $x x x$ & $\begin{array}{l}0,35 \\
(0,183-0,686)\end{array}$ \\
\hline \multicolumn{4}{|l|}{ Número de consultas de pré-natal } \\
\hline Pelo menos 6 & $x x x$ & $x x x$ & Ref. \\
\hline Menos de 6 & $x x x$ & $x x x$ & $\begin{array}{l}1,87 \\
(0,827-4,240)\end{array}$ \\
\hline Teste Interação Exames* Consultas & $x x x$ & $x x x$ & p-valor: 0,926 \\
\hline Pseudo R2 & $2,1 \%$ & $5,5 \%$ & $11,6 \%$ \\
\hline Qualidade do ajuste & $x x x$ & $x x x$ & p-valor: 0,993 \\
\hline
\end{tabular}

Modelo 1 (componentes distais). Modelo 2 (componentes distais e intermediários). Modelo 3 (componentes distais, intermediários e proximais).

RP - razão de prevalência. IC - intervalo de confiança. Ref. - referência (valor = 1).

A baixa escolaridade tem sido repetidamente associada à sífilis na gestação e à sífilis congênita. No presente estudo, o risco ajustado de ter um bebê com sífilis congênita quase dobrou entre mulheres com ensino fundamental incompleto, comparando com aquelas com ensino fundamental completo ou mais. A importância do nível educacional foi enfatizada em análise que incluiu mais de 23 mil mulheres. ${ }^{11}$ Embora a categoria de referência (ensino superior completo) tenha sido diferente da que foi por nós adotada, vale assinalar que a magnitude do baixo nível educacional aumentou em 16 vezes o desfecho sífilis congênita no estudo citado.

Uma pesquisa que enfocou casos de sífilis congênita detectados na maternidade estudada mostrou taxas de 24,0/1.000 nascidos vivos, 20,7/1.000 nascidos vivos e 20,6/1.000 nascidos vivos em 2002, 2003 e 2004 respectivamente. ${ }^{11}$ Os autores verificaram que mais de
$50 \%$ (107/161) das mães de bebês com sífilis congênita receberam assistência pré-natal no hospital-sede do estudo. Este cenário aponta para o fato de o fenômeno da sífilis congênita não estar esgotado na referida unidade de saúde, bem como em Niterói e adjacências.

Depois do ajustamento do modelo final, foi possível verificar que enquanto o pré-natal em Niterói foi um achado estatisticamente significante e reduziu o risco do desfecho de sífilis congênita em $52 \%$, a análise englobando o pré-natal em unidades do PMF, sem discriminar a localização das unidades por municípios, não revelou significância estatística e não permaneceu no modelo final.

O PMF de Niterói, lançado em 1992, manteve como diretriz a vinculação da clientela às equipes assistenciais, servindo de inspiração para o programa Estratégia de Saúde da Família do Ministério da Saúde. ${ }^{12}$ A análise 
categorizada que considerou o pré-natal fornecido por esse modelo nas unidades de Niterói e de outros municípios conjuntamente versus outro modelo qualquer não refletiu em redução de risco de sífilis congênita, evidenciando que a gravidade do problema deve ser tratada regionalmente.

Como preconiza a OMS, ${ }^{7}$ desenvolver ações intersetoriais com a participação de outros ministérios, além do da saúde, de programas de organizações não governamentais dirigidos à saúde reprodutiva e controle das infecções sexualmente transmissíveis etc. são fundamentais para o controle da doença em cenários de recursos escassos.

Outro achado do presente estudo foi o efeito do número de testes de sífilis realizados. Mães internadas na maternidade que tiveram em seus históricos pelo menos três testes para detectar sífilis apresentaram uma redução de $65 \%$ do risco de ter bebês com sífilis congênita, comparadas àquelas que fizeram menos de três exames. Tais achados se mantiveram estatisticamente significativos mesmo após o ajustamento do modelo final. Ainda que Domingues e Leal ${ }^{10}$ não tenham encontrado significância estatística em relação ao número de testes, as autoras mostraram que os testes durante a gestação tendem a reduzir o desfecho sífilis congênita, considerando se um único exame ou se dois exames foram independentemente realizados. Assim, os resultados do presente estudo reforçam a importância do terceiro exame no período gestacional, para a eliminação da sífilis congênita por meio de diagnóstico e de tratamento da doença.

Nos últimos anos, a sífilis congênita vem ganhando mais atenção das autoridades em todo o mundo. Ainda que ela não tenha sido diretamente incluída entre os Objetivos de Desenvolvimento do Milênio, no ano $2000,{ }^{13}$ reafirmados em $2015,{ }^{14}$ as metas de redução da mortalidade infantil, de melhoria da saúde materna e de combate a algumas doenças, entre as quais o HIV/ AIDS, compreendem medidas que alcançam também a sífilis. ${ }^{15}$ Reconhecendo as dificuldades para prevenir a aquisição da infecção, a OMS elaborou um documento voltado para a eliminação da sífilis congênita, focando: i) no compromisso político; ii) no acesso e qualidade dos serviços de saúde; iii) na triagem e tratamento de gestantes e parceiros; e no iv) fortalecimento das medidas de vigilância, monitoramento e avaliação. ${ }^{7}$

O cenário no Brasil favorece a consolidação dos quatro pilares estratégicos propostos pela OMS visando à eliminação da sífilis congênita. Há políticas públicas de saúde de âmbito nacional em pleno funcionamento no país, como:

a) o Sistema Único de Saúde (SUS);

b) o fortalecimento da Atenção Básica;

c) a humanização do pré-natal, do parto e do puerpério;

d) e a implantação da Rede Cegonha.

A cobertura de pré-natal é praticamente universal e conta com a oferta de testes de triagem e de diagnóstico de diferentes tipos. O tratamento medicamentoso é disponibilizado na rede de serviços públicos de saúde, incluindo os da Atenção Básica, com protocolos clínicos bem-delineados..$^{16}$ Além disso, a sífilis congênita faz parte da lista de doenças de notificação compulsória desde 1986. ${ }^{17}$ A vigilância epidemiológica, o monitoramento e a avaliação são facilitados via informações do Sistema de Informação de Agravos de Notificação (Sinan), um subsistema criado em 1993 e regulamentado em 1998 especificamente para lidar com a vigilância de doenças de notificação compulsória. ${ }^{18}$

\section{CONCLUSÕES}

Embora o presente estudo tenha se concentrado em aspectos que vêm sendo analisados em estudos de sífilis congênita, destaca-se como vantagem a implementação das análises, considerando relações teórico-hierárquicas e não apenas relações estatísticas.

Concluindo, a caracterização de mães de bebês com sífilis congênita delineada no presente estudo incluiu aspectos difíceis de controlar a curto prazo, como foi o caso da baixa escolaridade. No entanto, questões de competência de gestores e de profissionais de saúde mostraram-se determinantes para o controle dessa infecção. O enfoque no município de realização do pré-natal sugere que investimentos para melhorar a qualidade desse serviço, com cumprimento do protocolo de consultas, de testes para sífilis e de tratamento completo e oportuno devem ser priorizados. 


\section{REFERÊNCIAS}

1 Ministério da Saúde. Secretaria de Vigilância em Saúde. Departamento de Vigilância, Prevenção e Controle das Doenças Sexualmente Transmissíveis, AIDS e Hepatites Virais. Manual técnico para diagnóstico da sífilis [acesso em 9 dez 2018]. Brasília: MS; 2016. Disponível em: http://www.aids.gov.br/ pt-br/pub/2016/manual-tecnico-para-diagnostico-da-sifilis

2 Wijesooriya NS, Rochat RW, Kamb ML, Turlapati P, Temmerman M, Broutet N et al. Global burden of maternal and congenital syphilis in 2008 and 2012: a health systems modelling study. Lancet Glob Health. 2016;4(8):e525-33. DOI: https://doi.org/10.1016/S2214-109X(16)30135-8

3 Newman L, Kamb M, Hawkes S, Gomez G, Say L, Seuc A et al. Global estimates of syphilis in pregnancy and associated adverse outcomes: analysis of multinational antenatal surveillance data. Plos Med. 2013;10(2):el001396. DOI: https://doi.org/10.1371/journal.pmed.1001396

4 Qin J, Yang T, Xiao S, Tan H, Feng T, Fu H. Reported estimates of adverse pregnancy outcomes among women with and without syphilis: a systematic review and meta-analysis. Plos One. 2014;9(7):el02203. DOI: https://doi.org/10.1371/journal. pone.0102203

5 Secretaria de Estado de Saúde do Rio de Janeiro. Secretaria de Vigilância em Saúde. Superintendência de Vigilância Epidemiológica e Ambiental. Gerência de DST-AIDS e Hepatites Virais. Informe epidemiológico: sífilis materna e congênita. n. 1/2016. Rio de Janeiro: Vigilância das DST-AIDS; 2016.

6 World Health Organization. Baseline report on global sexually transmitted infection surveillance 2012 [accessed on Dez 9, 2018]. Geneva: WHO Press; 2013. Available from: https://www.who.int/reproductivehealth/publications/ rtis/9789241505895/en/

7 World Health Organization. The global elimination of congenital syphilis: rationale and strategy for action [accessed on Dez 9, 2018]. Geneva: WHO Press; 2007. Available from: https://www.who.int/reproductivehealth/publications/ rtis/9789241595858/en/

8 Victora CG, Huttly SR, Fuchs SC, Olinto MT. The role of conceptual frameworks in epidemiological analysis: a hierarchical approach. Int J Epidemiol. 1997;26(1):224-7. DOI: https://doi.org/10.1093/ije/26.1.224

9 Magalhães DM, Kawaguchi IA, Dias A, Calderon I de M. Sífilis materna e congênita: ainda um desafio. Cad Saúde Pública. 2013;29(6):1109-20. http://dx.doi.org/10.1590/ S0102-311X2013000600008
10 Domingues RM, Leal Mdo C. Incidência de sífilis congênita e fatores associados à transmissão vertical da sífilis: dados do estudo Nascer no Brasil. Cad Saúde Pública. 2016;32(6):e00082415. http://dx.doi. org/10.1590/0102-311X00082415

11 Schetini J, Ferreira DC, Passos MRL, Salles EB, Santos DDG, Rapozo DCM. Estudo da prevalência de sífilis congênita em um hospital da rede sus de Niterói - RJ [acesso em 9 dez 2018]. DST - J Bras Doenças Sex Transm. 2005;17(1):1823. Disponível em: http://www.dst.uff.br/revistal7-1-2005/ estudodaprevalencia.pdf

12 Hubner LCM, Franco TB. O programa médico de família de Niterói como estratégia de implementação de um modelo de atenção que contemple os princípios e diretrizes do SUS [acesso em 9 dez 2018]. Physis. 2007;17(1):173-91. Disponível em: http://www.scielo.br/pdf/physis/v17n1/v17nlal0.pdf

13 United Nations. 55/2 United Nations Millennium Declaration. Resolution adopted by the General Assembly. Fifty-fifth session. 18 Sep, 2000 [accessed on Oct 14, 2017]. Available from: http://www.un.org/millennium/declaration/ares552e. pdf

14 United Nations. The Millennium Development Goals Report 2015 [accessed on Oct 13, 2017. New York: UN; 2015. Available from: http://www.un.org/millenniumgoals/2015_MDG_ Report/pdf/MDG\%202015\%20rev\%20(July\%201).pdf

15 World Health Organization. Methods for surveillance and monitoring of congenital syphilis elimination within existing systems [accessed on Dez 9, 2018]. Geneva: WHO Press; 2011. Available from: https://www.who.int/reproductivehealth/ publications/rtis/9789241503020/en/

16 Ministério da Saúde. Secretaria de Vigilância em Saúde. Departamento de DST, AIDS e Hepatites Virais. Protocolo clínico e diretrizes terapêuticas para atenção integral às pessoas com infecções sexualmente transmissíveis. Brasília: MS; 2015.

17 Secretaria de Estado da Saúde de São Paulo. Secretaria de Vigilância Epidemiológica. Coordenação do Programa estadual DST/AIDS-SP. Coordenadoria de Controle de Doenças [acesso em 9 dez 2018]. Sífilis congênita e sífilis na gestação. Rev Saúde Pública. 2008;42(4):768-72. Disponível em: http://www. scielo.br/pdf/rsp/v42n4/itss.pdf

18 Ministério da Saúde. Secretaria de Vigilância em Saúde. Departamento de Vigilância Epidemiológica. Sistema de Informação de Agravos de Notificação: normas e rotinas [acesso em 9 dez 2018]. Brasília: MS; 2006. Disponível em: http://bvsms.saude.gov.br/bvs/publicacoes/sistema_ informacao_agravos_notificacao_sinan.pdf 